\title{
Momentum Broadening of a Fast Parton in a Perturbative Quark-Gluon Plasma
}

\author{
Abhijit Majumder \\ Department of Physics, The Ohio State University, Columbus, OH 43210, USA \\ Berndt Müller \\ Department of Physics, Duke University, Durham, NC 27708, USA \\ Stanisław Mrówczyński \\ Institute of Physics, Jan Kochanowski University, 25-406 Kielce, Poland and \\ Sottan Institute for Nuclear Studies, 00-681 Warsaw, Poland
}

(Dated: November 17, 2009)

\begin{abstract}
The average transverse momentum transfer per unit path length to a fast parton scattering elastically in a perturbative quark-gluon plasma is related to the radiative energy loss of the parton. We first calculate the momentum transfer coefficient $\hat{q}$ in terms of a classical Langevin problem and then define it quantum-mechanically through scattering matrix element. After treating the well known case of a quark-gluon plasma in equilibrium we consider an off-equilibrium unstable plasma. As a specific example, we treat the two-stream plasma with unstable modes of longitudinal chromoelectric field. In the presence of the instabilities, $\hat{q}$ is shown to exponentially grow in time.
\end{abstract}

\section{INTRODUCTION}

When a highly energetic parton travels through dense QCD matter, it receives random kicks from elastic interactions with constituents of the plasma. The average transverse momentum transfer per unit path length is related to the radiative energy loss of the parton [1]. The parameter describing the average amount of transverse momentum broadening per unit length is called $\hat{q}$ and is defined as

$$
\hat{q} \equiv d\left\langle\Delta \mathbf{p}_{T}^{2}\right\rangle / d z
$$

when the fast parton flies along the direction $z$. The values of $\hat{q}$ extracted from experimental data on relativistic heavy-ion collisions vary in a rather broad range $0.5-15 \mathrm{GeV}^{2} / \mathrm{fm}$ depending on the model of hard particle propagation in strongly interacting matter produced in nuclear collisions [2, 3].

The calculations of $\hat{q}$ for the case of perturbative quarkgluon plasma in equilibrium are well understood (see [4, 5] for recent work). For such a plasma the value of $\hat{q}$ is predicted to lie at the lower end of the range of values deduced from experiments [3]. However, it is not at all obvious that the quark-gluon plasma produced in a heavy ion collision is in equilibrium during the whole time interval of the propagation of the fast parton. It is rather expected that the momentum distribution of plasma partons is anisotropic at the early stage of relativistic heavy-ion. And recently $\hat{q}$ has been computed [6, 7] for the case of homogenous quark-gluon plasma with anisotropic momentum distribution. However, such a plasma is unstable due to the chromomagnetic modes (for a review see [8]). The fact that unstable systems are explicitly time dependent as unstable modes exponentially grow in time has not been taken into account in the previous analytical calculations [6, 7]. However, numerical simulations [9, 10] indicate that $\hat{q}$ receives a sizable contribution from these unstable modes. Also, possible phenomenological consequences of the momentum broadening effect of an unstable plasma on hard partons have been proposed [9, 11].

In this paper we develop the approach suggested in [12] (see also [13]) to study the transverse momentum fluctuations in terms of classical Langevin problem. We calculate $\hat{q}$ by treating the parton as an energetic classical particle with $\mathrm{SU}(3)$ color charge moving in the presence of the fluctuating color fields. Then, $\hat{q}$ is expressed through the correlation function of chromodynamic fields computed in [14], or equivalently, through by the unordered gluon propagator in Hard Thermal Loop (HTL) approximation. For the equilibrium plasma the Langevin approach recovers the known result obtained within the standard thermal field theory [15]. We then apply the Langevin approach to the case of a parton traversing an off-equilibrium unstable plasma. For the sake of simplicity, we study the two-stream system and consider only the contribution from the unstable modes of longitudinal chromoelectric field, neglecting the contribution from transverse fields. We show that $\hat{q}$ grows exponentially in time due to the exponential growth of the unstable mode amplitude.

\section{CLASSICAL APPROACH}

We consider a classical parton which moves across a quark-gluon plasma. Its motion is described by the Wong equations [16]

$$
\begin{aligned}
& \frac{d x^{\mu}(\tau)}{d \tau}=u^{\mu}(\tau), \\
& \frac{d p^{\mu}(\tau)}{d \tau}=g Q^{a}(\tau) F_{a}^{\mu \nu}(x(\tau)) u_{\nu}(\tau),
\end{aligned}
$$




$$
\frac{d Q_{a}(\tau)}{d \tau}=-g f^{a b c} p_{\mu}(\tau) A_{b}^{\mu}(x(\tau)) Q_{c}(\tau),
$$

where $\tau, x^{\mu}(\tau), u^{\mu}(\tau)$ and $p^{\mu}(\tau)$ are, respectively, the parton's proper time, its trajectory, four-velocity and four-momentum; $F_{a}^{\mu \nu}$ and $A_{a}^{\mu}$ denote the chromodynamic field strength tensor and four-potential, respectively, and $Q^{a}$ is the classical color charge of the parton.

We look for a solution of the Wong equations in a specific gauge and the gauge dependence of our results is discussed a posteriori. We assume that the potential vanishes along the parton's trajectory by imposing the axial gauge condition $p_{\mu}(\tau) A_{a}^{\mu}(x(\tau))=0$. Then, Eq. (4) simply states that $Q_{a}$ is constant as a function of $\tau$.

We solve Eqs. (2. 3) assuming that the momentum of the parton is so high that the changes in its direction of motion caused by the interactions with the medium are small compared with the momentum of the parton: $|\Delta \mathbf{p}| /|\mathbf{p}| \ll 1$. The change in the velocity vector $\mathbf{v}$ of the parton are then negligible, and we can consider the parton to move along a straight-line path with constant velocity. If the parton is massless or nearly massless (a light quark or a gluon), its speed can be approximated by the speed of light. Although we assume further on that the fast parton is at least approximately massless, we note that the approximation of a straight line trajectory with constant velocity also applies to a heavy quark with mass $M$ and momentum such that $|\mathbf{p}|=\gamma M|\mathbf{v}| \gg|\Delta \mathbf{p}|$.

In the limit of massless particle, the proper time becomes a meaningless concept and we formally solve the equation of motion (3) to eliminate it. Then, we get

$$
p^{\mu}(\tau)=p^{\mu}(0)+g Q_{a} \int_{0}^{\tau} d \tau^{\prime} F_{a}^{\mu \nu}\left(x\left(\tau^{\prime}\right)\right) u_{\nu}\left(\tau^{\prime}\right) .
$$

In general, this is not an explicit solution of (3) as the four-velocity $u_{\nu}(\tau)$ on the right-hand side of (5) depends on the four-momentum. However, the fourvelocity, as explained above, is approximated by a constant: $u_{\nu}(\tau) \approx u_{\nu}(0)$. Assuming that the direction of motion of the parton is in the positive $x^{3}$-direction, we have explicitly $u^{\mu}=\gamma(1,0,0, v)$ with the Lorentz factor $\gamma=\left(1-v^{2}\right)^{-1 / 2}$. Then (5) becomes

$$
p^{\mu}(t)=p^{\mu}(0)+g Q_{a} \int_{0}^{t} d t^{\prime}\left(F_{a}^{\mu 0}\left(x^{\prime}\right)-v F_{a}^{\mu 3}\left(x^{\prime}\right)\right),
$$

where $x^{\prime} \equiv x\left(t^{\prime}\right)$ and $t=\gamma \tau$.

In the limit $v \rightarrow 1$, the form of (6) motivates the introduction of light-cone variables defined as

$$
p^{ \pm} \equiv \frac{p^{0} \pm p^{3}}{\sqrt{2}} .
$$

In the new coordinates the four-vector components are understood as $p^{\mu}=\left(p^{+}, p^{-}, p^{1}, p^{2}\right)$ and the product of two four-vectors is

$$
p^{\mu} q_{\mu}=p^{+} q^{-}+p^{-} q^{+}-p^{1} q^{1}-p^{2} q^{2} .
$$

Assuming that the parton's initial position is $\mathbf{x}(0)=0$, its trajectory is given by $x^{\mu}(t)=\sqrt{2}(t, 0,0,0)$, and Eq. (6) takes the form

$$
p^{\mu}\left(x^{+}\right)=p^{\mu}(0)+g Q_{a} \int_{0}^{x^{+}} d y^{+} F_{a}^{\mu-}\left(y^{+}\right) .
$$

Here $F_{a}^{\mu-}\left(y^{+}\right)$is a short-hand notation for $F_{a}^{\mu-}\left(x^{\nu}\left(y^{+}\right)\right)$ with $x^{\nu}\left(y^{+}\right)=\left(y^{+}, 0,0,0\right)$. If the parton moves in the negative $x^{3}$-direction, Eq. (9) changes to

$$
p^{\mu}\left(x^{-}\right)=p^{\mu}(0)+g Q_{a} \int_{0}^{x^{-}} d y^{-} F_{a}^{\mu+}\left(y^{-}\right) .
$$

In the following we shall assume that the parton travels in the positive $x^{3}$-direction.

In the spirit of the Langevin approach, we consider the ensemble average $\left\langle p^{\mu}\left(x^{+}\right) p^{\nu}\left(x^{+}\right)\right\rangle$indicated by the angular brackets. The ensemble average involves averaging over color charges. We recall that

$$
\int d Q Q_{a} Q_{b}=C_{2} \delta^{a b}
$$

where $C_{2}=1 / 2$ for particles (quarks) in fundamental representation of the $\mathrm{SU}\left(N_{c}\right)$ group and $C_{2}=N_{c}$ for particles (gluons) in adjoint representation. Then, we find

$$
\begin{aligned}
& \left\langle p^{\mu}\left(x^{+}\right) p^{\nu}\left(x^{+}\right)\right\rangle=p^{\mu}(0) p^{\nu}(0) \\
& \quad+g^{2} C \int_{0}^{x^{+}} d y_{1}^{+} d y_{2}^{+}\left\langle F_{a}^{\mu-}\left(y_{1}^{+}\right) F_{a}^{\nu-}\left(y_{2}^{+}\right)\right\rangle,
\end{aligned}
$$

where the color factor $C$ is given as

$$
C \equiv\left\{\begin{array}{rlrl}
\frac{C_{2}}{N_{c}} & =\frac{1}{2 N_{c}} & & \text { (fundamental rep.) } \\
\frac{C_{2}}{N_{c}^{2}-1}=\frac{N_{c}}{N_{c}^{2}-1} & & \text { (adjoint rep.). }
\end{array}\right.
$$

It is related to the eigenvalue $C_{R}(R=F, A)$ of the quadratic Casimir operator as

$$
C_{R}=C\left(N_{c}^{2}-1\right) .
$$

Let us consider $\left\langle p^{\mu}\left(x^{+}\right) p_{\mu}\left(x^{+}\right)\right\rangle$in more detail:

$$
\begin{aligned}
& \left\langle p^{\mu}\left(x^{+}\right) p_{\mu}\left(x^{+}\right)\right\rangle=p^{\mu}(0) p_{\mu}(0) \\
& \quad+g^{2} C \int_{0}^{x^{+}} d y_{1}^{+} d y_{2}^{+} g_{\mu \nu}\left\langle F_{a}^{\mu-}\left(y_{1}^{+}\right) F_{a}^{\nu-}\left(y_{2}^{+}\right)\right\rangle .
\end{aligned}
$$

An interesting feature of (15) is that although $\mu$ runs over all four components $0,1,2,3$ or $+,-, 1,2$, only the transverse components $(\mu=1,2)$ contribute to $g_{\mu \nu}\left\langle F_{a}^{\mu-}\left(y_{1}^{+}\right) F_{a}^{\nu-}\left(y_{2}^{+}\right)\right\rangle$. This is easily seen by writing the expression out in light-cone coordinates and recalling that the antisymmetry of $F_{a}^{\alpha \beta}$ requires $F_{a}^{--}=0$. The terms for $\mu=+,-$ thus vanish and consequently,

$$
\begin{aligned}
& g_{\mu \nu} F_{a}^{\mu-}\left(y_{1}\right) F_{a}^{\nu-}\left(y_{2}\right)= \\
& \quad-F_{a}^{1-}\left(y_{1}\right) F_{a}^{1-}\left(y_{2}\right)-F_{a}^{2-}\left(y_{1}\right) F_{a}^{2-}\left(y_{2}\right) .
\end{aligned}
$$


The fact that only the transverse components $(\mu=1,2)$ contribute implies that we can write

$$
\begin{aligned}
& \left\langle\Delta \mathbf{p}_{T}^{2}\left(x^{+}\right)\right\rangle \equiv\left\langle\Delta \mathbf{p}_{T}\left(x^{+}\right) \cdot \Delta \mathbf{p}_{T}\left(x^{+}\right)\right\rangle \\
& \quad=-g^{2} C \int_{0}^{x^{+}} d y_{1}^{+} d y_{2}^{+} g_{\mu \nu}\left\langle F_{a}^{\mu-}\left(y_{1}^{+}\right) F_{a}^{\nu-}\left(y_{2}^{+}\right)\right\rangle,
\end{aligned}
$$

where $\Delta \mathbf{p}_{T}\left(x^{+}\right)=\mathbf{p}_{T}\left(x^{+}\right)-\mathbf{p}_{T}(0)$.

Equation (17), which is the main result of this section, has been derived in a specific gauge and the righthand side of the equation is, in general, gauge dependent. The gauge independence can be restored by inserting the Wilson line between the fields $F_{a}^{\mu-}\left(y_{1}^{+}\right)$and $F_{a}^{\nu-}\left(y_{2}^{+}\right)$in the correlation function $\left\langle F_{a}^{\mu-}\left(y_{1}^{+}\right) F_{a}^{\nu-}\left(y_{2}^{+}\right)\right\rangle$. However, the correlation functions, which are further used in our study, are computed in the Hard Loop approximation. These functions appear to be gauge independent even without the Wilson-line insertion, as discussed in detail in Sec. VIIIA of [14]. Therefore, Eq. (17) is gauge independent within the approximations we apply.

We now assume that the quark-gluon plasma is translationally invariant in space and time and, hence, the correlator $\left\langle F_{a}^{\mu-}\left(y_{1}^{+}\right) F_{a}^{\nu-}\left(y_{2}^{+}\right)\right\rangle$depends only on the difference $y_{1}^{+}-y_{2}^{+}$. This assumption, which is relevant for equilibrium plasmas, will be not adopted for the twostream system discussed in Sec. VI as the growth of unstable modes break the translational invariance in time. Making use of the translational invariance, we introduce the fluctuation spectrum

$$
\left\langle F_{a}^{\mu-} F_{a}^{\nu-}\right\rangle_{k} \equiv \int d^{4} x e^{i k x}\left\langle F_{a}^{\mu-}(x) F_{a}^{\nu-}(0)\right\rangle,
$$

which allows us to express Eq. (17) as

$$
\begin{aligned}
& \left\langle\Delta \mathbf{p}_{T}^{2}\left(x^{+}\right)\right\rangle=-g^{2} C \int_{0}^{x^{+}} d y_{1}^{+} d y_{2}^{+} \\
& \quad \times \int \frac{d^{4} k}{(2 \pi)^{4}} e^{i k^{-}\left(y_{1}^{+}-y_{2}^{+}\right)} g_{\mu \nu}\left\langle F_{a}^{-\mu} F_{a}^{\nu-}\right\rangle_{k} .
\end{aligned}
$$

Since the double integral over $y_{1}^{+}$and $y_{2}^{+}$tends to a delta function of $k^{-}$in the long-time limit

$$
\begin{aligned}
\int_{0}^{x^{+}} d & y_{1}^{+} d y_{2}^{+} e^{i k^{-}\left(y_{1}^{+}-y_{2}^{+}\right)} \\
& =\frac{4 \sin ^{2}\left(k^{-} x^{+} / 2\right)}{\left(k^{-}\right)^{2}} \stackrel{x^{+} \rightarrow \infty}{\longrightarrow} 2 \pi x^{+} \delta\left(k^{-}\right),
\end{aligned}
$$

we can define the transport coefficient

$$
\begin{aligned}
\hat{q} & \equiv \lim _{t \rightarrow \infty} \frac{1}{t}\left\langle\Delta \mathbf{p}_{T}^{2}(t)\right\rangle=\lim _{x^{+} \rightarrow \infty} \frac{\sqrt{2}}{x^{+}}\left\langle\Delta \mathbf{p}_{T}^{2}\left(x^{+}\right)\right\rangle \\
& =-2 \sqrt{2} \pi g^{2} C \int \frac{d^{4} k}{(2 \pi)^{4}} \delta\left(k^{-}\right) g_{\mu \nu}\left\langle F_{a}^{\mu-} F_{a}^{\nu-}\right\rangle_{k},
\end{aligned}
$$

which was introduced in Eq. (11) as the transverse momentum broadening per unit path length.

From here on we can proceed in two different ways. We can either use the fluctuation spectra of chromodynamic fields derived in [14], or we can express $\left\langle F_{a}^{\mu-} F_{a}^{\nu-}\right\rangle_{k}$ through the unordered gluon propagator in the HTL approximation. In the two following sections we pursue both approaches. The first one is shorter, while the second one allows us to relate our results to those obtained in [7].

\section{FLUCTUATION SPECTRA OF CHROMODYNAMIC FIELDS}

The fluctuation spectra of the equilibrium plasma, as computed in [14], are

$$
\begin{aligned}
\left\langle E_{a}^{i} E_{b}^{j}\right\rangle_{k} & =2 \delta^{a b} T \omega^{3}\left[\frac{k^{i} k^{j}}{\mathbf{k}^{2}} \frac{\Im \varepsilon_{L}(\omega, \mathbf{k})}{\left|\omega^{2} \varepsilon_{L}(\omega, \mathbf{k})\right|^{2}}\right. \\
& \left.+\left(\delta^{i j}-\frac{k^{i} k^{j}}{\mathbf{k}^{2}}\right) \frac{\Im \varepsilon_{T}(\omega, \mathbf{k})}{\left|\omega^{2} \varepsilon_{T}(\omega, \mathbf{k})-\mathbf{k}^{2}\right|^{2}}\right] \\
\left\langle B_{a}^{i} B_{b}^{j}\right\rangle_{k} & =2 \delta^{a b} T \omega \mathbf{k}^{2}\left(\delta^{i j}-\frac{k^{i} k^{j}}{\mathbf{k}^{2}}\right) \\
& \times \frac{\Im \varepsilon_{T}(\omega, \mathbf{k})}{\left|\omega^{2} \varepsilon_{T}(\omega, \mathbf{k})-\mathbf{k}^{2}\right|^{2}} \\
\left\langle B_{a}^{i} E_{b}^{j}\right\rangle_{k} & =\left\langle E_{a}^{j} B_{b}^{i}\right\rangle_{k}=2 \delta^{a b} T \omega^{2} \epsilon^{i k j} k^{k} \\
& \times \frac{\Im \varepsilon_{T}(\omega, \mathbf{k})}{\left|\omega^{2} \varepsilon_{T}(\omega, \mathbf{k})-\mathbf{k}^{2}\right|^{2}}
\end{aligned}
$$

where $k=(\omega, \mathbf{k}), \epsilon^{i j k}$ is the antisymmetric tensor, $T$ is the system's temperature and $\varepsilon_{L, T}(\omega, \mathbf{k})$ are choromodielectric functions which for the equilibrium plasma of massless particles are well-known to be [15]

$$
\begin{aligned}
& \left.\Re \varepsilon_{L}(\omega, \mathbf{k})=1+\frac{m_{D}^{2}}{\mathbf{k}^{2}}\left[1-\frac{\omega}{2|\mathbf{k}|} \ln \mid \frac{\omega+|\mathbf{k}|}{\omega-|\mathbf{k}|}\right]\right], \\
& \Im \varepsilon_{L}(\omega, \mathbf{k})=\frac{\pi}{2} \Theta\left(\mathbf{k}^{2}-\omega^{2}\right) \frac{m_{D}^{2} \omega}{|\mathbf{k}|^{3}}, \\
& \Re \varepsilon_{T}(\omega, \mathbf{k})=1-\frac{m_{D}^{2}}{2 \mathbf{k}^{2}}\left[1-\frac{\omega^{2}-\mathbf{k}^{2}}{2 \omega|\mathbf{k}|} \ln \left|\frac{\omega+|\mathbf{k}|}{\omega-|\mathbf{k}|}\right|\right], \\
& \Im \varepsilon_{T}(\omega, \mathbf{k})=\frac{\pi}{4} \Theta\left(\mathbf{k}^{2}-\omega^{2}\right) \frac{m_{D}^{2}\left(\mathbf{k}^{2}-\omega^{2}\right)}{\omega|\mathbf{k}|^{3}},
\end{aligned}
$$

where $m_{D}$ is the Debye mass:

$$
m_{D}^{2}=\frac{g^{2} T^{2}}{6}\left(N_{f}+2 N_{c}\right) .
$$

Since

$$
\begin{aligned}
-g_{\mu \nu}\left\langle F_{a}^{\mu-} F_{a}^{\nu-}\right\rangle_{k} & =\left\langle E_{a}^{x} E_{a}^{x}\right\rangle_{k}+\left\langle E_{a}^{y} E_{a}^{y}\right\rangle_{k} \\
& -\left\langle E_{a}^{x} B_{a}^{y}\right\rangle_{k}+\left\langle E_{a}^{y} B_{a}^{x}\right\rangle_{k} \\
& -\left\langle B_{a}^{y} E_{a}^{x}\right\rangle_{k}+\left\langle B_{a}^{x} E_{a}^{y}\right\rangle_{k} \\
& +\left\langle B_{a}^{x} B_{a}^{x}\right\rangle_{k}+\left\langle B_{a}^{y} B_{a}^{y}\right\rangle_{k},
\end{aligned}
$$


Equation (21) yields

$$
\begin{aligned}
\hat{q}= & 2 g^{2} C_{R} T \int \frac{d^{3} k}{(2 \pi)^{3}} \frac{k_{T}^{2}}{k_{z} \mathbf{k}^{2}} \\
& \times\left[\frac{\Im \varepsilon_{L}\left(k_{z}, \mathbf{k}\right)}{\left|\varepsilon_{L}\left(k_{z}, \mathbf{k}\right)\right|^{2}}+\frac{k_{z}^{2} k_{T}^{2} \Im \varepsilon_{T}\left(k_{z}, \mathbf{k}\right)}{\left|k_{z}^{2} \varepsilon_{T}\left(k_{z}, \mathbf{k}\right)-\mathbf{k}^{2}\right|^{2}}\right] .
\end{aligned}
$$

The classical formula (27) holds for the inverse wave vectors of the fields which are much longer than the de Broglie wavelength of plasma particles. It requires $|\mathbf{k}| \ll T$ where $T$ is the plasma temperature. For larger wave vectors a quantum approach is needed which is discussed in Sec. V

Keeping in mind that the equations

$$
\varepsilon_{L}(\omega, \mathbf{k})=0, \quad \omega^{2} \varepsilon_{T}(\omega, \mathbf{k})-\mathbf{k}^{2}=0,
$$

determine the longitudinal and transverse collective modes in the isotropic plasma, one sees that the transverse momentum broadening, as given by the formula (27), is determined by the interaction of the fast parton with plasma collective excitations.

\section{GLUON PROPAGATOR}

As already mentioned, $\left\langle F_{a}^{\mu-} F_{a}^{\nu-}\right\rangle_{k}$, which enters Eq. (21), can be expressed through the unordered gluon propagator in the HTL approximation.

Neglecting all non-Abelian contributions, the Fourier transform of the field strength tensor is expressed through the four-potential as

$$
F_{a}^{\mu \nu}(k)=-i k^{\mu} A_{a}^{\nu}(k)+i k^{\nu} A_{a}^{\mu}(k),
$$

allowing us to rewrite (21) as

$$
\hat{q}=\sqrt{2} \pi g^{2} C \int \frac{d^{4} k}{(2 \pi)^{4}} \delta\left(k^{-}\right) k_{T}^{2}\left\langle A_{a}^{-} A_{a}^{-}\right\rangle_{k} .
$$

Here we made use of the relation $\partial^{0} \pm \partial^{3}=\sqrt{2} \partial^{\mp}$ and further used the fact that only the $\mu=1,2$ components contribute in the field strength correlator. We also dropped all terms proportional to $k^{-}$in view of the delta function.

Because $y_{1}^{+}$and $y_{2}^{+}$in Eq. (17) run independently from 0 to $x^{+}$, the correlator $\left\langle A_{a}^{-} A_{a}^{-}\right\rangle_{k}$ is the Fourier transform of the time-unordered gluon Green function. Since the plasma of interest is on average color neutral, the gluon propagators such as $\left\langle A_{a}^{\mu}(x) A_{b}^{\nu}(y)\right\rangle$ are proportional to $\delta^{a b}$. We drop for now the color indices but when the gluon propagator $\left\langle A_{a}^{-} A_{a}^{-}\right\rangle_{k}$ is substituted in Eq. (29), the coefficient $\delta^{a a}=N_{c}^{2}-1$ will included.

For translationally invariant systems, one defines the time-unordered $(>,<)$ Green functions

$$
\begin{aligned}
& i D_{\mu \nu}^{>}(x-y) \equiv\left\langle A_{\mu}(x) A_{\nu}(y)\right\rangle, \\
& i D_{\mu \nu}^{<}(x-y) \equiv\left\langle A_{\nu}(y) A_{\mu}(x)\right\rangle,
\end{aligned}
$$

where, as already mentioned, the color indices are suppressed. We also introduce the spectral function

$$
\begin{aligned}
\rho_{\mu \nu}(x-y) & =\left\langle\left[A_{\mu}(x), A_{\nu}(y)\right]\right\rangle \\
& =i D_{\mu \nu}^{>}(x-y)-i D_{\mu \nu}^{<}(x-y) .
\end{aligned}
$$

The equilibrium Green functions $D_{\mu \nu}^{>}(x), D_{\mu \nu}^{<}(x)$ obey the Kubo-Martin-Schwinger condition

$$
D_{\mu \nu}^{>}(t, \mathbf{x})=D_{\mu \nu}^{<}(t+i \beta, \mathbf{x}),
$$

or

$$
D_{\mu \nu}^{>}(k)=e^{\beta \omega} D_{\mu \nu}^{<}(k),
$$

where $\beta \equiv 1 / T$. Combining the definition (32) and the KMS condition (34), we have

$$
\begin{aligned}
& i D_{\mu \nu}^{<}(k)=n(\omega) \rho_{\mu \nu}(k), \\
& i D_{\mu \nu}^{>}(k)=(n(\omega)+1) \rho_{\mu \nu}(k),
\end{aligned}
$$

where $n(\omega) \equiv\left(e^{\beta \omega}-1\right)^{-1}$ denotes the Bose distribution.

The spectral function in the HTL approximation can be expressed as [15]

$$
\begin{aligned}
\rho_{\mu \nu}(k)= & \frac{2 \Im \varepsilon_{L}(\omega, \mathbf{k})}{k^{2}\left|\varepsilon_{L}(\omega, \mathbf{k})\right|^{2}} P_{\mu \nu}^{L}(k) \\
& +\frac{2 \omega^{2} \Im \varepsilon_{T}(\omega, \mathbf{k})}{\left|\omega^{2} \varepsilon_{T}(\omega, \mathbf{k})-\mathbf{k}^{2}\right|^{2}} P_{\mu \nu}^{T}(k),
\end{aligned}
$$

with the projectors defined as

$$
\begin{aligned}
P_{T}^{i j}(k) & =\delta^{i j}-\frac{k^{i} k^{j}}{\mathbf{k}^{2}}, \quad P_{T}^{0 \mu}(k)=0, \\
P_{L}^{\mu \nu}(k) & =-g^{\mu \nu}+\frac{k^{\mu} k^{\nu}}{k^{2}}-P_{T}^{\mu \nu}(k) .
\end{aligned}
$$

We identify the correlator $\left\langle A_{a}^{-} A_{b}^{-}\right\rangle_{k}$ from Eq. (21) with the time-unordered Green function $D_{>}^{--}(k)$. Substituting $D_{>}^{--}(k)$ given by Eqs. (35, 37) into Eq. (29), we obtain

$$
\begin{aligned}
\hat{q}= & 4 \sqrt{2} \pi g^{2} C_{R} \int \frac{d^{4} k}{(2 \pi)^{4}} \delta\left(k^{-}\right) n(\omega) k_{T}^{2} \\
& \times\left[\frac{\Im \varepsilon_{L}(\omega, \mathbf{k})}{k^{2}\left|\varepsilon_{L}(\omega, \mathbf{k})\right|^{2}} P_{L}^{--}(k)\right. \\
& \left.+\frac{\omega^{2} \Im \varepsilon_{T}(\omega, \mathbf{k})}{\left|\omega^{2} \varepsilon_{T}(\omega, \mathbf{k})-\mathbf{k}^{2}\right|^{2}} P_{T}^{--}(k)\right],
\end{aligned}
$$

where $C_{R}$ is the eigenvalue of the quadratic Casimir operator given by Eq. (14).

With the help of formulas (38, 39) we find

$$
\begin{aligned}
& P_{T}^{--}(k)=\frac{k_{T}^{2}}{2 \mathbf{k}^{2}}, \\
& P_{L}^{--}(k)=\frac{k^{-} k^{-}}{\mathbf{k}^{2}}-\frac{k_{T}^{2}}{2 \mathbf{k}^{2}},
\end{aligned}
$$


and thus, we finally obtain

$$
\begin{aligned}
\hat{q}= & 2 g^{2} C_{R} \int \frac{d^{3} k}{(2 \pi)^{3}} n\left(k_{z}\right) \frac{k_{T}^{2}}{\mathbf{k}^{2}} \\
& \times\left[\frac{\Im \varepsilon_{L}\left(k_{z}, \mathbf{k}\right)}{\left|\varepsilon_{L}\left(k_{z}, \mathbf{k}\right)\right|^{2}}+\frac{k_{z}^{2} k_{T}^{2} \Im \varepsilon_{T}\left(k_{z}, \mathbf{k}\right)}{\left|k_{z}^{2} \varepsilon_{T}\left(k_{z}, \mathbf{k}\right)-\mathbf{k}^{2}\right|^{2}}\right],
\end{aligned}
$$

where $k_{z} \equiv k^{3}$. In the classical field limit, where $n(\omega) \approx$ $T / \omega$, we obtain again the expression (27).

Since the HTL approximation holds for $|\mathbf{k}| \ll T$, the integration in Eq. (43), as well as in the fully classical formula (27), should be cut off at $\left|\mathbf{k}_{\max }\right| \ll T$. For larger wave vectors we need a quantum approach, which is discussed in the next section.

\section{QUANTUM APPROACH}

Here we sketch the derivation of $\hat{q}$ following the strategy outlined in [15] for the electromagnetic energy loss of a fast muon. We present how to obtain two contributions to $\hat{q}$ coming from soft $(|\mathbf{q}| \ll T)$ and hard $(|\mathbf{q}| \sim T)$ momentum transfers. We discuss only the main steps of the derivation as the procedure is mostly described in the literature [4, 7]. This section is included for completeness of our study.

Within a quantum approach $\hat{q}$ is defined as

$$
\hat{q} \equiv \int d^{2} q_{T} q_{T}^{2} \frac{d^{2} \Gamma}{d^{2} q_{T}}
$$

where $\Gamma$ is the rate (probability per unit time) for elastic collisions of parton with plasma particles. For a fast parton with four-momentum $p_{1}$ which scatters on plasma constituents of species $i$ with four-momenta $p_{2}$, the rate corresponding to the binary process $p_{1}+p_{2} \rightarrow p_{1}^{\prime}+p_{2}^{\prime}$ is given as

$$
\begin{aligned}
\Gamma= & \frac{1}{E_{1}} \int \frac{d^{3} p_{1}^{\prime}}{2 E_{1}^{\prime}(2 \pi)^{3}} \frac{d^{3} p_{2}^{\prime}}{2 E_{2}^{\prime}(2 \pi)^{3}} \frac{d^{3} p_{2}}{E_{2}(2 \pi)^{3}} \\
& \times(2 \pi)^{4} \delta^{(4)}\left(p_{1}+p_{2}-p_{1}^{\prime}-p_{2}^{\prime}\right) \\
& \times \sum_{i} f_{i}\left(\mathbf{p}_{2}\right)\left[1 \pm f_{i}\left(\mathbf{p}_{2}^{\prime}\right)\right]\left|\mathcal{M}_{i}\left(p_{1}, p_{2} ; p_{1}^{\prime}, p_{2}^{\prime}\right)\right|^{2}
\end{aligned}
$$

where $f_{i}(\mathbf{p})$ is the distribution function of particles of species $i$ and $\mathcal{M}_{i}\left(p_{1}, p_{2} ; p_{1}^{\prime}, p_{2}^{\prime}\right)$ is the scattering matrix element. The definition of $\left|\mathcal{M}_{i}\right|^{2}$ involves summation over final spins and colors and an average over the initial spin and color of the fast parton.

The matrix element of parton-parton scattering is assumed to be dominated by the one-gluon exchange process. Then, the matrix element squared can be expressed as the self energy of the fast quark. When the fast parton is a massless quark, one finds [7]

$$
\hat{q}=\frac{1}{4 E} \operatorname{Tr}\left[\not p \Sigma_{\mathrm{w}}^{>}(p)\right]
$$

where $\Sigma_{\mathrm{w}}^{>}(p)$ is the fast quark self-energy 'weighted' with $q_{T}^{2}$. It is given as

$$
\Sigma_{\mathrm{w}}^{>}(p)=g^{2} C_{F} \int \frac{d^{3} p^{\prime}}{2 E^{\prime}(2 \pi)^{3}} q_{T}^{2} \gamma^{\mu} \not p^{\prime} \gamma^{\nu} D_{\mu \nu}^{<}(q),
$$

where $p^{\prime}=p+q$ and $D_{\mu \nu}^{<}(q)$ is the gluon propagator (stripped of the color factor $\delta^{a b}$ ) which includes the oneloop correction. Changing the orientation of the momentum $q$, the propagator $D_{\mu \nu}^{<}(q)$ (35) should be replaced by $D_{\mu \nu}^{>}(q)$ (36) but we get the same result as $n\left(-q_{0}\right)+1=-n\left(q_{0}\right)$.

In the soft domain, where $|\mathbf{q}| \ll T$, the one-loop gluon propagator $D_{\mu \nu}^{<}(k)$ is obtained in the HTL approximation. Substituting $D_{\mu \nu}^{<}(k)$ provided by Eqs. (35. 37] into Eq. (46), we find the differential rate which enters Eq. (44) as [17]

$$
\begin{aligned}
& \frac{d^{2} \Gamma_{\mathrm{soft}}}{d^{2} q_{T}}=2 g^{2} C_{R} \int^{\Lambda} \frac{d q_{z}}{(2 \pi)^{3}} \frac{n\left(q_{z}\right)}{\mathbf{q}^{2}} \\
& \times\left[\frac{\Im \varepsilon_{L}\left(q_{z}, \mathbf{q}\right)}{\left|\varepsilon_{L}\left(q_{z}, \mathbf{q}\right)\right|^{2}}+\frac{q_{z}^{2} q_{T}^{2} \Im \varepsilon_{T}\left(q_{z}, \mathbf{q}\right)}{\left|q_{z}^{2} \varepsilon_{T}\left(q_{z}, \mathbf{k}\right)-\mathbf{q}^{2}\right|^{2}}\right],
\end{aligned}
$$

where the upper cut-off $\Lambda$ obeys $g T<\Lambda<T$. We have here replaced $C_{F}$ by $C_{R}$ as a similar analysis can be performed for fast gluons. The expression for $\hat{q}$ given by Eq. (44) with (48) agrees, as expected, with Eq. (43). By explicit evaluation one shows [4] that the soft collision rate (48) can be approximated as

$$
\frac{d \Gamma_{\text {soft }}}{d^{2} q_{T}} \approx g^{2} \frac{C_{R}}{(2 \pi)^{2}} \frac{T m_{D}^{2}}{q_{T}^{2}\left(q_{T}^{2}+m_{D}^{2}\right)} .
$$

The hard $(|\mathbf{q}| \sim T)$ contribution to $\hat{q}$ can be found directly from Eqs. (44,45) using the unscreened gluon propagator. The approximate result is [4]

$$
\frac{d \Gamma_{\text {hard }}}{d^{2} q_{T}} \approx g^{4} \frac{C_{R}}{(2 \pi)^{2}} \frac{\rho}{q_{T}^{4}}
$$

where $\rho$ is the properly normalized parton density in the plasma.

The complete result for $\hat{q}$ is obtained by summing up the hard and soft contributions. An arbitrary parameter $\Lambda$, which separates the soft momentum transfers from the hard one, is the ultraviolet cut-off for the soft contribution and the infrared cut-off for the hard contribution. $\Lambda$ is eliminated from the final answer, as the soft contribution depends logarithmically on $\Lambda$ and the hard contribution depends logarithmically on $\Lambda^{-1}$. Since the hard contribution also depends logarithmically on the ultraviolet bound provided by two-body kinematics of the scattering process, we can simply extrapolate the soft contribution to the hard domain with no significant error [4]. In conclusion, the integral in the soft contribution to $\hat{q}$, as given by Eqs. (4449), can be extended to the maximal momentum transfer $q_{T}^{\max }$ or $k_{T}^{\max }$ roughly equal $\sqrt{E T}$ where $E=p^{0}$ is the energy of the fast parton. 


\section{TWO-STREAM UNSTABLE PLASMA}

Our aim here is to calculate the momentum broadening of a fast parton in unstable anisotropic plasmas. As mentioned in the Introduction, this problem was studied previously [6, 7] in a way similar to that presented in Sec. V] Namely, the hard thermal loop gluon propagator of an equilibrium plasma was replaced by the hard loop propagator of an anisotropic plasma [18]. Such an approach, however, does not take into account that unstable systems are intrinsically time dependent because the unstable modes grow exponentially in time. We show here how these modes influence the momentum broadening of a fast parton. For the sake of analytical tractability, we do not consider the anisotropic plasma with a momentum distribution likely to be found in the early stage of rela- tivistic heavy-ion collisions, as the correlation functions of chromodynamic fields are difficult to obtain for such a plasma. Instead, we consider a fast parton in the more tractable, but also unstable two-stream plasma. With this simplified example we hope to elucidate the general features of the problem of momentum broadening in unstable plasmas.

The distribution function of the two-stream plasma is chosen to be of the form

$$
f(\mathbf{p})=(2 \pi)^{3} n\left[\delta^{(3)}\left(\mathbf{p}-\mathbf{p}_{0}\right)+\delta^{(3)}\left(\mathbf{p}+\mathbf{p}_{0}\right)\right],
$$

where $n$ is the effective parton density in a single stream. The distribution function (51) should be treated as an idealization of the two-peak distribution where the particles have momenta close to $\mathbf{p}_{0}$ or $-\mathbf{p}_{0}$.

The two-stream plasma is unstable with respect to both longitudinal and transverse modes. Because the twostream plasma does not have a direct phenomenological application, and we are only interested in showing the effect of unstable modes on $\hat{q}$ in principle, we consider for simplicity that the parton interacts only with the longitudinal electric fields. It is worth noting that there exist also unstable transverse modes, which will have a similar effect on $\hat{q}$. The correlation function of the longitudinal field components was derived in [14]. We are mostly interested in the exponentially growing modes but the correlation function which represents only such modes becomes meaningless when the growth rate tends to zero. Therefore, we denote the correlation function with the subscript label "exp" to indicate that it includes both, the exponentially growing and the exponentially decaying modes. The expression for the correlation function reads [14]:

$$
\begin{aligned}
\left\langle E_{a}^{i}\left(t_{1}, \mathbf{r}_{1}\right) E_{b}^{j}\left(t_{2}, \mathbf{r}_{2}\right)\right\rangle_{\exp } & =\frac{g^{2}}{2} \delta^{a b} n \int \frac{d^{3} k}{(2 \pi)^{3}} \frac{e^{i \mathbf{k}\left(\mathbf{r}_{1}-\mathbf{r}_{2}\right)}}{\mathbf{k}^{4}} \frac{k^{i} k^{j}}{\left(\omega_{+}^{2}-\omega_{-}^{2}\right)^{2}} \frac{\left(\gamma_{\mathbf{k}}^{2}+(\mathbf{k} \cdot \mathbf{u})^{2}\right)^{2}}{\gamma_{\mathbf{k}}^{2}} \\
& \times\left[\left(\gamma_{\mathbf{k}}^{2}+(\mathbf{k} \cdot \mathbf{u})^{2}\right) \cosh \left(\gamma_{\mathbf{k}}\left(t_{1}+t_{2}\right)\right)+\left(\gamma_{\mathbf{k}}^{2}-(\mathbf{k} \cdot \mathbf{u})^{2}\right) \cosh \left(\gamma_{\mathbf{k}}\left(t_{1}-t_{2}\right)\right)\right]
\end{aligned}
$$

where $\mathbf{u} \equiv \mathbf{p}_{0} / E_{\mathbf{p}_{0}}$ is the stream velocity and $\pm \omega_{ \pm}(\mathbf{k})$ are the four roots of the dispersion equation $\varepsilon_{L}(\omega, \mathbf{k})=0$ :

$$
\omega_{ \pm}^{2}(\mathbf{k})=\frac{1}{\mathbf{k}^{2}}\left[\mathbf{k}^{2}(\mathbf{k} \cdot \mathbf{u})^{2}+\mu^{2}\left(\mathbf{k}^{2}-(\mathbf{k} \cdot \mathbf{u})^{2}\right) \pm \mu \sqrt{\left(\mathbf{k}^{2}-(\mathbf{k} \cdot \mathbf{u})^{2}\right)\left(4 \mathbf{k}^{2}(\mathbf{k} \cdot \mathbf{u})^{2}+\mu^{2}\left(\mathbf{k}^{2}-(\mathbf{k} \cdot \mathbf{u})^{2}\right)\right)}\right]
$$

with $\mu^{2} \equiv g^{2} n / 2 E_{\mathbf{p}_{0}} . \gamma_{\mathbf{k}}$ is the instability growth rate $\left(0<\gamma_{\mathbf{k}} \in \mathbb{R}\right)$ defined as $\omega_{-}(\mathbf{k})=i \gamma_{\mathbf{k}}$. The integration over $\mathbf{k}$ in Eq. (52) is performed over the domain of unstable longitudinal modes defined by the requirement

$$
\mathbf{k} \cdot \mathbf{u} \neq 0 \text { and } \mathbf{k}^{2}(\mathbf{k} \cdot \mathbf{u})^{2}<2 \mu^{2}\left(\mathbf{k}^{2}-(\mathbf{k} \cdot \mathbf{u})^{2}\right)
$$

The correlation function (52) is obviously invariant with respect to space translations - it depends on the difference $\left(\mathbf{r}_{1}-\mathbf{r}_{2}\right)$ only. The initial plasma state is on average homogeneous and it remains so over the course of time. The time dependence of the correlation function (52) is very different from the space dependence, and it is not invariant under time translations. The electric fields grow exponentially and so does the correlation function, both in the variables $\left(t_{1}+t_{2}\right)$ and $\left(t_{1}-t_{2}\right)$. The fluctuation spectrum also evolves in time as the growth rate of unstable modes is wave vector dependent. After a sufficiently long time the fluctuation spectrum is dominated by the fastest growing modes.

Substituting the correlation function (52) into Eq. (15) and reverting to integration over time $t$ instead of the light-cone variable $y^{+}$, we find 


$$
\begin{aligned}
\left\langle\Delta \mathbf{p}_{T}^{2}(t)\right\rangle & =g^{2} C \int_{0}^{t} d t^{\prime} \int_{0}^{t} d t^{\prime \prime}\left[\left\langleE _ { a } ^ { x } \left(t^{\prime}, \mathbf{r}\left(t^{\prime}\right) E_{a}^{x}\left(t^{\prime \prime}, \mathbf{r}\left(t^{\prime \prime}\right)\right\rangle+\left\langle E_{a}^{y}\left(t^{\prime}, \mathbf{r}\left(t^{\prime}\right) E_{a}^{y}\left(t^{\prime \prime}, \mathbf{r}\left(t^{\prime \prime}\right)\right\rangle\right]\right.\right.\right.\right. \\
& =\frac{g^{4}}{4} C_{R} n \int \frac{d^{3} k}{(2 \pi)^{3}} \frac{k_{T}^{2}}{\mathbf{k}^{4}\left(\omega_{+}^{2}-\omega_{-}^{2}\right)^{2}} \frac{\left(\gamma_{\mathbf{k}}^{2}+(\mathbf{k} \cdot \mathbf{u})^{2}\right)^{2}}{\gamma_{\mathbf{k}}^{2}\left(k_{z}^{2}+\gamma_{\mathbf{k}}^{2}\right)} \\
& \times\left[\left(\gamma_{\mathbf{k}}^{2}+(\mathbf{k} \cdot \mathbf{u})^{2}\right)\left(\left|e^{\left(i k_{z}+\gamma_{\mathbf{k}}\right) t}-1\right|^{2}+\left|e^{\left(i k_{z}-\gamma_{\mathbf{k}}\right) t}-1\right|^{2}\right)+4\left(\gamma_{\mathbf{k}}^{2}-(\mathbf{k} \cdot \mathbf{u})^{2}\right) \frac{k_{z}^{2}-\gamma_{\mathbf{k}}^{2}}{k_{z}^{2}+\gamma_{\mathbf{k}}^{2}}\right] .
\end{aligned}
$$

Taking into account only the fastest growing contribution to Eq. (55), we obtain

$$
\hat{q}=\frac{d\left\langle\Delta \mathbf{p}_{T}^{2}(t)\right\rangle}{d t} \approx \frac{g^{4}}{2} C_{R} n \int \frac{d^{3} k}{(2 \pi)^{3}} e^{2 \gamma_{\mathbf{k}} t} \frac{k_{T}^{2}\left(\gamma_{\mathbf{k}}^{2}+(\mathbf{k} \cdot \mathbf{u})^{2}\right)^{3}}{\mathbf{k}^{4}\left(\omega_{+}^{2}-\omega_{-}^{2}\right)^{2} \gamma_{\mathbf{k}}\left(k_{z}^{2}+\gamma_{\mathbf{k}}^{2}\right)} .
$$

This expression diverges when $\gamma_{\mathbf{k}} \rightarrow 0$. It happens because only the unstable modes are taken into account. The formula given in Eq. (55) remains finite in the limit $\gamma_{\mathbf{k}} \rightarrow 0$. The momentum broadening (56) grows exponentially in time as the spontaneously growing fields exert an exponentially growing influence on the propagating parton. This effect is missing in the previous results [6, 7] obtained for the unstable anisotropic plasma, which was treated as a stationary medium.

The calculation of the momentum broadening carried out here does not assume that the medium is close to equilibrium or that the growth rate of the instabilities is small enough to invoke the static approximation. Even though we have restricted our calculation to the simple and analytically tractable case of the two stream system, the underlying formalism may be applied to calculate the momentum broadening in an arbitrary medium as long as the field strength correlators are calculable.

The differences between the results obtained in this manuscript and those in previous articles using a steady state approach are two-fold: An unstable system is not time-translation invariant and thus a calculation based on time translation invariance is in general invalid. When the propagator depends independently on $t$ and $t^{\prime}$, rather than on the difference $t-t^{\prime}$, one obtains a function of two frequency variables when performing the (one-sided) Fourier transformations with respect to the two time variables.

In assuming that the gluon occupation number $n(\omega)$ is a function of only a single frequency, authors of Refs. [6, 7] implicitly assume that the rate of instability growth is slow enough so that one may impose approxi- mate time translation invariance over certain time periods. Note, however, that even if we ignore the $\left(t_{1}+t_{2}\right)$ dependence in the first term of the correlator (52), the Fourier integral with respect to $t_{1}-t_{2}$ of the second term does not exist because of the exponential divergence. If we were to ignore this problem for the moment, we would obtain results which could be reconciled with those of Refs. [6, 7] only if we would make the additional assumption that the system would remain very close to thermal equilibrium. In the following, we illustrate this point in more detail by contrasting our calculation with the results obtained in the steady-state approach for the twostream system. It is useful to start from the expression (43) for the parameter $\hat{q}$.

In order to decompose the expression for $\hat{q}$ into a convolution of an occupation number and a spectral density we now assume approximate time translation invariance. We again restrict our consideration to the longitudinal response of the plasma. The calculation is most easily carried out in the temporal axial gauge, in which the spectral function of longitudinal electric fields is given by

$$
\rho_{L}^{i j}(k)=\frac{2 \Im \varepsilon_{L}(k)}{\omega^{2}\left|\varepsilon_{L}(k)\right|^{2}} \frac{k^{i} k^{j}}{\mathbf{k}^{2}}
$$

A straightforward calculation shows that this expression continues to hold when the system is anisotropic, but the anisotropy is determined by a single vector, here the flow velocity $\mathbf{u}$. The dielectric function $\varepsilon_{L}(\omega, \mathbf{k})$ for the two-stream system is given in Ref. [14]:

$$
\varepsilon_{L}(\omega, \mathbf{k})=1-\mu^{2} \frac{\mathbf{k}^{2}-(\mathbf{k} \cdot \mathbf{u})^{2}}{\mathbf{k}^{2}}\left[\frac{1}{(\omega-\mathbf{k} \cdot \mathbf{u})^{2}}+\frac{1}{(\omega+\mathbf{k} \cdot \mathbf{u})^{2}}\right]=\frac{\left(\omega^{2}-\omega_{+}(\mathbf{k})^{2}\right)\left(\omega^{2}-\omega_{-}(\mathbf{k})^{2}\right)}{\left(\omega^{2}-(\mathbf{k} \cdot \mathbf{u})^{2}\right)^{2}}
$$

where $\omega_{ \pm}(\mathbf{k})$ is given by (53). We note that the expression for $\Im \varepsilon_{L}(\omega, \mathbf{k})$ vanishes for real $\omega$ and $\mathbf{k}$ for the two-stream 
system. The expression $\Im \varepsilon_{L}(\omega, \mathbf{k}) /\left|\varepsilon_{L}(\omega, \mathbf{k})\right|^{2}$ in (57) should therefore be replaced by

$$
-\Im\left[\frac{1}{\varepsilon_{L}(\omega, \mathbf{k})+i \operatorname{sgn}(\omega) 0^{+}}\right]=\pi \operatorname{sgn}(\omega) \delta\left(\varepsilon_{L}(\omega, \mathbf{k})\right) .
$$

The expression analogous to (55) derived from the steady-state formula (43) then reads:

$$
\begin{aligned}
\hat{q} & =-2 \pi g^{2} C_{R} \int \frac{d^{4} k}{(2 \pi)^{4}} n(\omega, \mathbf{k}) \delta\left(\omega-k_{z}\right) \frac{k_{T}^{2}}{\mathbf{k}^{2}} \Im\left[\frac{1}{\varepsilon_{L}\left(k_{z}, \mathbf{k}\right)+i \operatorname{sgn}\left(k_{z}\right) 0^{+}}\right] . \\
& =\pi g^{2} C_{R} \int \frac{d^{3} k}{(2 \pi)^{3}} n\left(k_{z}, \mathbf{k}\right) \frac{k_{T}^{2}}{\mathbf{k}^{2}} \operatorname{sgn}\left(k_{z}\right) \delta\left(\varepsilon_{L}\left(k_{z}, \mathbf{k}\right)\right) .
\end{aligned}
$$

Here $n(\omega, \mathbf{k})$ is the average occupation number of the soft gluon modes. For the case of a medium in thermal equilibrium, $n(\omega, \mathbf{k})$ is given by the Bose distribution and for soft frequencies may be approximated by $T / \omega$. For the case of a medium that is barely anisotropic, i. e., where the corrections to the Bose distribution are small, one may persist with this form for the occupation number for short periods of time. This is the approximation made in Refs. [6, 7]. Such an approximation will, no doubt, break down as the medium continues to depart from isotropic equilibrium or as the unstable modes grow with time. Besides the insistence on the static approximation, the choice of the equilibrium Bose distribution without specifying the short range of time over which this is applicable, constitutes an additional source of error in these earlier works.

In order to obtain the correct form of the occupation number for any time, in the limit that one may still invoke the static approximation, one needs to recalculate the two unordered space-time propagators,

$$
\begin{aligned}
& D^{>}(t)=\sum_{n}\langle n|\hat{\rho} A(t) A(0)| n\rangle, \\
& D^{<}(t)=\sum_{n}\langle n|\hat{\rho} A(0) A(t)| n\rangle,
\end{aligned}
$$

for the specific density operator characteristic to the unstable system being considered. In the equation above, $A(t)$ is the vector potential of the glue field suppressing Lorentz and color indices, as well as space variables. In this case one may obtain the spectral density (32) in momentum space and thus calculate the time dependent average occupation number as

$$
n(\omega)=\frac{D^{<}(\omega)}{D^{>}(\omega)-D^{<}(\omega)} .
$$

Only in the case that the density operator is given by the thermal Gibbs expression $\hat{\rho}=\exp [-\beta \hat{H}]$, will there be a simple Kubo-Martin-Schwinger (KMS) relation (34) resulting in the Bose distribution for the occupation number. For any other form of the density operator, the occupation number may be considerably different from the Bose distribution, even under the assumption of approximate time translation invariance.

As a result, we find that the use of a static approximation and a Bose distribution for the occupation number of the unstable modes restricts the applicability of the calculations of Ref. [6, 7] to very short times in a plasma where the growth rates of the instabilities are rather small. It should come as no surprise that in such a regime, the effect of such modes on the momentum broadening of a hard parton is minimal. In an arbitrary weakly coupled quark-gluon plasma with a large momentum anisotropy, the growth rate of the unstable modes may be large and, as a result, the calculations in the current article remain the only consistent approach to calculating the broadening of a hard parton traversing such a system. Furthermore, we note that the badly singular contributions encountered by the authors of Ref. [6, 7] in their calculations presumably arise from the improper formulation of the problem as explained above.

\section{SUMMARY AND OUTLOOK}

We have studied the effect of color field fluctuations in a QCD medium on the propagation of a hard parton. In the first part of this work, we described a classical Langevin approach to compute the transport coefficient $\hat{q}$ which denotes the amount of transverse momentum broadening per unit path length of an energetic parton. In this Langevin approach the coefficient is expressed through the correlation function of chromodynamic fields along the light-cone or, equivalently, through the unordered gluon propagator. In the case of a perturbative equilibrium plasma when the field correlation functions or the gluon propagators are described in the HTL approximation we reproduced the known result [4, 7].

In the second part of this study we considered a hard parton in an off-equilibrium, unstable plasma. Our explicit calculations performed for the two-stream plasma showed that unstable modes with exponentially growing amplitude cause an exponential growth of the transverse momentum broadening. Our result demonstrates that the quasi-static approximation used in previous calculations of the transport coefficient $\hat{q}$ is not applicable.

It should be clearly stated, however, that the temporal evolution of anisotropic plasma is not the only feature which needs to be taken into account when transport properties of the unstable plasma are studied. When the plasma is populated with strong fields, new inter- 
action mechanisms appear. For example, it has been recently argued that in a spontaneously chromomagnetized plasma the viscosity acquires an anomalous contribution [19] while synchrotron radiation contributes to the parton energy loss [20, 21].

The formalism for the calculation of $\hat{q}$ used in this work is applicable to numerical simulations. It would be interesting to perform such a calculation for the case of a realistic, three-dimensional quark-gluon plasma off equilibrium. We note that the calculations of the field correlators are very complex even in the linear response regime [14]. When the growth of a continuum of unstable modes results in strong nonlinear interactions among them, as demonstrated in the case of the anisotropic plasma by numerical simulations [22, 23], an analytical treatment of the field correlators seems to be impossible. In view of the great phenomenological importance of the transport coefficient $\hat{q}$ in relativistic heavy ion physics, a numerical evaluation of the field correlator in an unstable, anisotropic plasma would be very desirable. According to the exploratory simulations [9, 10], $\hat{q}$ can be significantly larger in the unstable plasma than in an equilibrium plasma.

Another promising direction for future study is the extension of our formalism to momentum broadening in a strongly coupled plasma. Due to the asymptotic freedom of quantum chromodynamics, a highly virtual, energetic parton can be weakly coupled to the quark-gluon plasma, even if the dynamics of the plasma itself is governed by strong coupling. Such an investigation would, for example, pursue the question of potentially large logarithmic corrections to $\hat{q}$ arising from large ratios of scales, such as $Q^{2} / T^{2}$, where $Q^{2}$ denotes the virtuality of the hard parton. Instead of relying on an expansion in orders of the coupling constant $g$, an approach based on factorization into nonperturbative matrix elements of the medium and perturbative dynamics of the parton itself would lend itself to a renormalization group treatment [24]. Although $\hat{q}$ would not be calculable $a b$ initio in such an approach, its scale evolution with the energy or virtuality of the fast parton could be predicted.

\section{Acknowledgments}

We thank Rolf Baier for helpful correspondence. This work was supported in part by the U.S. Department of Energy under grant numbers DE-FG02-05ER41367 and DE-FG02-01ER41190. St.M. thanks the members of the QCD theory group at Duke University for their warm hospitality during his visit.
[1] R. Baier, Y. L. Dokshitzer, A. H. Mueller, S. Peigne and D. Schiff, Nucl. Phys. B 484, 265 (1997).

[2] K. J. Eskola, H. Honkanen, C. A. Salgado and U. A. Wiedemann, Nucl. Phys. A 747, 511 (2005).

[3] R. Baier and D. Schiff, JHEP 0609, 059 (2006).

[4] P. Arnold and W. Xiao, Phys. Rev. D 78, 125008 (2008).

[5] S. Peigne and A. V. Smilga, Phys. Usp. 52, 659 (2009).

[6] P. Romatschke, Phys. Rev. C 75, 014901 (2007).

[7] R. Baier and Y. Mehtar-Tani, Phys. Rev. C 78, 064906 (2008).

[8] St. Mrówczyński, Acta Phys. Polon. B 37, 427 (2006).

[9] A. Dumitru, Y. Nara, B. Schenke and M. Strickland, Phys. Rev. C 78, 024909 (2008).

[10] B. Schenke, M. Strickland, A. Dumitru, Y. Nara and C. Greiner, Phys. Rev. C 79, 034903 (2009)

[11] A. Majumder, B. Müller and S. A. Bass, Phys. Rev. Lett. 99, 042301 (2007).

[12] A. Majumder and B. Müller, Phys. Rev. C 77, 054903 (2008).

[13] Z. T. Liang, X. N. Wang and J. Zhou, Phys. Rev. D 77,
$125010(2008)$

[14] St. Mrówczyński, Phys. Rev. D 77, 105022 (2008).

[15] M. Le Bellac, Thermal Field Theory (Cambridge University Press, Cambridge, 2000), Section 8.1.

[16] S. K. Wong, Nuovo Cim. A 65, 689 (1970).

[17] X. N. Wang, Phys. Lett. B 485, 157 (2000)

[18] St. Mrówczyński, A. Rebhan and M. Strickland, Phys. Rev. D 70, 025004 (2004).

[19] M. Asakawa, S. A. Bass and B. Müller, Phys. Rev. Lett. 96, 252301 (2006); Prog. Theor. Phys. 116, 725 (2007).

[20] E. V. Shuryak and I. Zahed, Phys. Rev. D 67, 054025 (2003).

[21] B. G. Zakharov, JETP Lett. 88, 475 (2008).

[22] P. Arnold and G. D. Moore, Phys. Rev. D 73, 025006 (2006).

[23] A. Dumitru, Y. Nara and M. Strickland, Phys. Rev. D 75, 025016 (2007).

[24] A. Majumder, arXiv:0901.4516 [nucl-th]. 